\title{
Elevated Thyroid Autoantibodies Aggravate Stroke Severity in Euthyroidism with Acute Ischemic Stroke
}

\author{
Jingyi Li $\mathbb{D}^{1},{ }^{1}$ Shoulong Hu, ${ }^{2}$ Fang Liu, ${ }^{3}$ Dapeng Wu, ${ }^{3}$ Wei Song, ${ }^{3}$ and Miao Hui ${ }^{3}$ \\ ${ }^{1}$ The Department of Endocrinology, Beijing United Family Hospital, Beijing, China \\ ${ }^{2}$ The Department of Ophthalmology, Beijing Children's Hospital, Capital Medical University, Beijing, China \\ ${ }^{3}$ The Department of Neurology, The First Affiliated Hospital, Tsinghua University, Beijing, China \\ Correspondence should be addressed to Jingyi Li; doctorlijingyi@163.com
}

Received 19 November 2021; Accepted 1 February 2022; Published 26 February 2022

Academic Editor: Ting Su

Copyright (C) 2022 Jingyi Li et al. This is an open access article distributed under the Creative Commons Attribution License, which permits unrestricted use, distribution, and reproduction in any medium, provided the original work is properly cited.

Introduction. Studies have indicated that immune reactions contribute to endothelial dysfunction and atherosclerosis. It is unclear whether thyroid dysfunction or elevated thyroid autoantibodies are associated with atherosclerosis. Therefore, we investigated the influence of thyroid autoimmunity related to elevated thyroid autoantibodies on functional outcome in euthyroidism with acute ischemic stroke (AIS). Methods. All patients with AIS underwent tests for thyroid function and thyroid antibodies (thyroid peroxidase antibody and thyroglobulin autoantibody). We divided the patients suffering from euthyroidism and AIS into positive thyroid autoantibody and negative thyroid autoantibody groups. Demographic profiles, risk factors, and functional outcomes were compared between the two groups. Results. Out of the total 422 patients, 50 (11.8\%) were included in the positive thyroid autoantibody group. The National Institutes of Health Stroke Scale (NIHSS) score at admission and discharge was higher in the positive thyroid autoantibody group than the negative thyroid autoantibody group $(P<0.05)$. In addition, there was significant difference in the mortality during hospitalizations between the two groups $(P<0.01)$. Conclusion. This study showed that thyroid autoantibodies aggravate stroke severity in euthyroidism with AIS. We speculate that vascular damage related to thyroid autoimmunity may aggravate the increased risk of unfavorable outcomes, independent of thyroid function.

\section{Introduction}

Ischemic stroke is one of the leading causes of morbidity and mortality worldwide, and endothelial dysfunction and arteriosclerosis are its primary etiologies. Several factors such as age, smoking, obesity, hypertension, diabetes, and dyslipidemia have been recognized as aggravating the progress of arteriosclerosis. In addition, in recent years, some studies have highlighted that the immune responses are profoundly involved in the progression of endothelial dysfunction and arteriosclerosis [1]. Several cases have provided evidence for the association between thyroid diseases and arterial damage $[2,3]$. A study showed the correlation between hyperthyroidism and intracranial arterial stenosis in stroke patients [4]. In addition, thyroid dysfunction and positive thyroid autoantibodies were shown in pediatric patients with moyamoya disease [5]. It is unclear whether thyroid dys- function or positive thyroid autoantibodies were associated with vascular diseases. Therefore, we retrospectively analyzed our AIS patients with euthyroidism and compared the stroke severity between the two groups with or without elevated thyroid peroxidase antibody (TPO-Ab) and thyroglobulin autoantibody $(\mathrm{Tg}-\mathrm{Ab})$ in China.

\section{Methods}

2.1. Patient Selection. We followed the methods of Cho et al. [6]. We retrospectively analyzed our AIS patients with euthyroidism admitted to the First Affiliated Hospital of Tsinghua University from January 2017 to December 2018 and compared the stroke severity between these patients with or without elevated TPO-Ab and Tg-Ab. The protocols of our study had been approved by the Tsinghua University ethics committee. The inclusion criteria were AIS confirmed 
by magnetic resonance imaging (MRI) within 3 days of symptom onset, and the results of thyroid function, TPO$\mathrm{Ab}$, and $\mathrm{Tg}-\mathrm{Ab}$ were available. Patients with an overt history of thyroid disease or diagnosed with thyroid disorder during admission; those with immunological, infectious, and toxic diseases; those undergoing thrombolytic treatment; and those on immunosuppressant drugs or immunomodulators were excluded. Thyroid function was considered based on thyroid-stimulating hormone (TSH) level. Of the initial 469 patients, 47 were excluded because of hyperthyroidism ( TSH $<0.27 \mathrm{mU} / \mathrm{L}, \quad n=6,1.28 \%$ ) and hypothyroidism $(\mathrm{TSH}>4.2 \mathrm{mU} / \mathrm{L}, n=41,8.74 \%)$. Finally, a total of 422 (TSH $0.27-4.2 \mathrm{mU} / \mathrm{L}, 89.98 \%$ ) patients with normal thyroid function were eligible for inclusion in this study.

2.2. Assessment of Clinical Courses. The baseline demographics and some vascular risk factors were collected. We especially paid more attention to the effects of aspirin. Patients were considered as being on aspirin therapy if they regularly took the drug within the 3-month period before admission. The patients were diagnosed with diabetes mellitus if the fasting blood glucose was elevated $(\geq 7.0 \mathrm{mmol} / \mathrm{L})$ at least 2 times or if patients were on regular hypoglycemic agents. Patients were diagnosed with hypertension if their blood pressure was elevated (systolic: $\geq 140 \mathrm{mmHg}$ or diastolic: $\geq 90 \mathrm{mmHg}$ ) or if they were on regular antihypertensive medication. The patients were diagnosed with hyperlipidemia if their fasting serum total cholesterol $(\geq 6.2 \mathrm{mmol} / \mathrm{L})$ or lowdensity lipoprotein cholesterol $(\geq 4.1 \mathrm{mmol} / \mathrm{L})$ was elevated, or if they were on regular lipid-lowering medication. Patients were considered smokers if they smoked at least one cigarette per day within the 3-month period before admission.

Laboratory tests included blood glucose, blood lipid profile, erythrocyte sedimentation rate, high-sensitivity C-reactive protein, fibrinogen, homocysteine, thyroid function, TPO-Ab, and $\mathrm{Tg}-\mathrm{Ab}$ in consecutive patients with AIS presenting within 3 days of symptom onset. The fasting blood samples were collected in the morning after admission. The thyroid functions were evaluated by measuring the serum levels of total triiodothyronine (TT3), free triiodothyronine (FT3), total thyroxine (TT4), free thyroxine (FT4), and thyroid- stimulating hormone (TSH). The reference ranges for TT3, FT3, TT4, FT4, and TSH, respectively, were 1.3$3.1 \mathrm{nmol} / \mathrm{L}, 2.8-7.1 \mathrm{pmol} / \mathrm{L}, 66-181 \mathrm{nmol} / \mathrm{L}, 12-22 \mathrm{pmol} / \mathrm{L}$, and $0.27-4.2 \mathrm{mU} / \mathrm{L}$. For the assessment of thyroid autoimmunity, the serum concentrations of the TPO-Ab and Tg-Ab were checked using electrochemiluminescence with a commercial kit (Roche, Basel, Switzerland). We divided the AIS patients into two groups according to the levels of the thyroid autoantibodies. The positive thyroid autoantibody (PTA) group was defined as either $\mathrm{TPO}-\mathrm{Ab}>34 \mathrm{IU} / \mathrm{mL}$ and/or $\mathrm{Tg}-\mathrm{Ab}>$ $115 \mathrm{IU} / \mathrm{mL}$ in accordance with the manufacturer's reference. The negative thyroid autoantibody (NTA) group was defined as $\mathrm{TPO}-\mathrm{Ab} \leq 34 \mathrm{IU} / \mathrm{mL}$ and $\mathrm{Tg}-\mathrm{Ab} \leq 115 \mathrm{IU} / \mathrm{mL}$.

Stroke severity was assessed according to the National Institutes of Health Stroke Scale (NIHSS) score at admission and discharge. In addition, the mortality during hospitalizations and hospitalization time were used to evaluate the unfavorable outcome.
TABle 1: Comparison of demographic features in AIS and euthyroidism with and without elevated thyroid autoantibodies.

\begin{tabular}{lccc}
\hline & PTA $(n=50)$ & NTA $(n=372)$ & $P$ value \\
\hline $\begin{array}{l}\text { Age, years } \\
\begin{array}{l}\text { Female, } \\
n(\%)\end{array}\end{array}$ & $25(50.0)$ & $113(30.4)$ & $P<0.01^{* *}$ \\
$\begin{array}{l}\text { Hypertension, } \\
n(\%)\end{array}$ & $46(92.0)$ & $332(89.2)$ & 0.550 \\
$\begin{array}{l}\text { Diabetes, } \\
n(\%)\end{array}$ & $29(58.0)$ & $197(53.0)$ & 0.503 \\
$\begin{array}{l}\text { Hyperlipidemia, } \\
n(\%)\end{array}$ & $29(58.0)$ & $234(62.9)$ & 0.502 \\
$\begin{array}{l}\text { Cigarette smoking, } \\
n(\%)\end{array}$ & $11(22.0)$ & $115(30.9)$ & 0.192 \\
$\begin{array}{l}\text { Aspirin therapy, } \\
n(\%)\end{array}$ & $11(22.0)$ & $97(26.1)$ & 0.529 \\
\hline
\end{tabular}

Data presented as mean \pm standard deviation or percentage. Abbreviations: AIS: acute ischemic stroke; PTA: positive thyroid autoantibody; NTA: negative thyroid autoantibody. ${ }^{*} P<0.05$ and ${ }^{* *} P<0.01$.

2.3. Statistical Analysis. Data were expressed as the mean \pm standard deviation or percentage. Statistical analysis was performed with SPSS (version 20.0; IBM Corporation, Armonk, NY, USA) between the two groups using independent sample two-tailed $t$-test and chi-square test. $P<0.05$ was considered to indicate statistically significant differences.

\section{Results}

Out of the included 422 patients, 50 (11.8\%) were included in the PTA group. Both the TPO-Ab and Tg-Ab were elevated in 22 patients in the PTA group. There was no significant difference on the prevalence of the positive TPO-Ab and the positive $\mathrm{Tg}-\mathrm{Ab}(n=37$ [8.8\%] vs. $n=34$ [8.1\%], $P=0.509$ ).

The baseline demographics (Table 1) and all the laboratory results including the risk factors related to the intracranial stenosis (Table 2) and the clinical features including the thyroid function (Table 3 ) of the PTA and NTA groups were shown in detail. Table 1 shows that more female patients had elevated thyroid autoantibodies $(n=25$ [50.0\%] vs. $n=113$ [30.4\%], $P<0.01)$ compared to the NTA group. The age and vascular risk factors were not significantly different between the two groups $(P>0.05)$. Furthermore, the rate of aspirin therapy was not significantly different between the two groups $(P>0.05)$ in Table 1 . Even though the protective high-density lipoprotein cholesterol was higher in the PTA group than the NTA group $(P<0.01)$ in Table 2 , the higher NIHSS scores at admission were observed in the PTA group than the NTA group $(P<0.05)$. The duration of hospital stay was similar between the two groups $(P=0.523)$. The NIHSS at discharge was evaluated again. Higher NIHSS scores at discharge could be used to predict poor prognosis and severity of AIS patients early in the PTA group than the NTA group in Table 3. Overall, seven patients died (four patients in the PTA group [8\%] and three patients in the NTA group $[0.8 \%](P<0.01))$. Therefore, there was significant difference 
TABLE 2: Comparison of the risk factors related to the intracranial stenosis in AIS and euthyroidism with and without elevated thyroid autoantibodies.

\begin{tabular}{|c|c|c|c|}
\hline & PTA $(n=50)$ & NTA $(n=372)$ & $P$ value \\
\hline \multicolumn{4}{|l|}{ Blood pressure at admission } \\
\hline Systolic blood pressure (mmHg) & $151.4 \pm 19.2$ & $149.2 \pm 21.5$ & 0.482 \\
\hline Diastolic blood pressure (mmHg) & $86.7 \pm 12.6$ & $85.0 \pm 13.8$ & 0.408 \\
\hline \multicolumn{4}{|l|}{ Blood lipid profile } \\
\hline Total cholesterol (mmol/L) & $4.57 \pm 1.06$ & $4.31 \pm 1.07$ & 0.118 \\
\hline Triglycerides (mmol/L) & $1.47 \pm 1.1$ & $1.54 \pm 1.02$ & 0.626 \\
\hline High-density lipoprotein cholesterol (mmol/L) & $1.33 \pm 0.41$ & $1.18 \pm 0.32$ & $P<0.01^{* *}$ \\
\hline Low-density lipoprotein cholesterol (mmol/L) & $3.0 \pm 1.02$ & $2.9 \pm 1.27$ & 0.571 \\
\hline \multicolumn{4}{|l|}{ Blood glucose profile } \\
\hline Fasting plasma glucose (mmol/L) & $7.21 \pm 3.44$ & $6.78 \pm 2.6$ & 0.296 \\
\hline Glycosylated hemoglobin (\%) & $7.0 \pm 2.3$ & $6.6 \pm 1.6$ & 0.238 \\
\hline Erythrocyte sedimentation rate $(\mathrm{mm} / \mathrm{h})$ & $10.6 \pm 8.4$ & $8.7 \pm 11.9$ & 0.33 \\
\hline High-sensitivity C-reactive protein (mg/L) & $10.31 \pm 17.8$ & $9.97 \pm 21.71$ & 0.92 \\
\hline Fibrinogen $(\mathrm{g} / \mathrm{L})$ & $3.18 \pm 0.77$ & $3.23 \pm 0.81$ & 0.685 \\
\hline Homocysteine (umol/L) & $16.26 \pm 12.67$ & $17.33 \pm 14.08$ & 0.618 \\
\hline
\end{tabular}

Data presented as mean \pm standard deviation or percentage. Abbreviations: AIS: acute ischemic stroke; PTA: positive thyroid autoantibody; NTA: negative thyroid autoantibody. ${ }^{*} P<0.05$ and ${ }^{* *} P<0.01$.

TABLE 3: Comparison of thyroid function and clinical features in AIS and euthyroidism with and without elevated thyroid autoantibodies.

\begin{tabular}{|c|c|c|c|}
\hline & PTA $(n=50)$ & NTA $(n=372)$ & $P$ value \\
\hline \multicolumn{4}{|l|}{ Thyroid function test } \\
\hline TT3 (pmol/L) & $1.67 \pm 0.33$ & $1.62 \pm 0.35$ & 0.350 \\
\hline FT3 (pmol/L) & $3.83 \pm 0.54$ & $4.02 \pm 0.74$ & $0.025^{*}$ \\
\hline TT4 (pmol/L) & $99.86 \pm 17.14$ & $96.39 \pm 21.94$ & 0.288 \\
\hline FT4 (pmol/L) & $16.33 \pm 2.6$ & $16.54 \pm 4.71$ & 0.753 \\
\hline TSH (mU/L) & $1.76 \pm 1.17$ & $1.68 \pm 0.92$ & 0.632 \\
\hline $\begin{array}{l}\text { NIHSS score } \\
\text { at admission }\end{array}$ & $5.3 \pm 5.4$ & $3.9 \pm 3.9$ & $0.025^{*}$ \\
\hline $\begin{array}{l}\text { NIHSS score } \\
\text { at discharge }\end{array}$ & $3.9 \pm 4.2$ & $2.8 \pm 3.1$ & $0.023^{*}$ \\
\hline Mortality, $n(\%)$ & $4(8 \%)$ & $3(0.8)$ & $P<0.01^{* *}$ \\
\hline $\begin{array}{l}\text { Duration of } \\
\text { hospital stay (days) }\end{array}$ & $14.3 \pm 7.1$ & $14.8 \pm 7.1$ & 0.523 \\
\hline
\end{tabular}

Data presented as mean \pm standard deviation or percentage. Abbreviations: AIS: acute ischemic stroke; PTA: positive thyroid autoantibody; NTA: negative thyroid autoantibody; TT3: total triiodothyronine; FT3: free triiodothyronine; TT4: total thyroxine; FT4: free thyroxine; TSH: thyroidstimulating hormone; NIHSS: National Institutes of Health Stroke Scale score. ${ }^{*} P<0.05$ and ${ }^{* *} P<0.01$.

in the mortality between the PTA group and NTA group in this study.

Table 3 shows that even though the mean values of TSH, TT3, TT4, FT3, and FT4 were all within the normal reference range, the FT3 level was significantly lower in AIS patients with elevated thyroid autoantibodies $(P<0.05)$.

\section{Discussion}

This study indicated that $\mathrm{TPO}-\mathrm{Ab}$ and $\mathrm{Tg}-\mathrm{Ab}$ aggravate stroke severity in euthyroidism with AIS, independent of thyroid function. In our study sample, we evaluated the possible influence of thyroid autoimmunity related to elevated $\mathrm{TPO}-\mathrm{Ab}$ and $\mathrm{Tg}-\mathrm{Ab}$ on functional outcome in euthyroidism with AIS in China. Some previous studies have indicated that autoimmune reactions were related to the occurrence of intracranial arterial stenosis in stroke patients with hyperthyroidism [4]. Hyperthyroidism was investigated as a risk factor for vascular damages related to poor functional outcome [7]. A case report detailed the intracranial stenosis and damages noticed in Graves' disease, which were relieved after glucocorticoid treatment and plasmapheresis [8]. The above several studies have shown that abnormal thyroid function may involve short- and long-term cardiovascular and cerebrovascular bad effects. However, it is unclear whether the autoimmunity related to elevated TPO-Ab or $\mathrm{Tg}-\mathrm{Ab}$ was associated with the severity of AIS. To exclude the effects of thyroid dysfunction on stroke functional outcome, we reviewed 422 patients with normal thyroid function and AIS. Some researchers have shown that male sex, diabetes, dyslipidemia, hypertension, smoking, and hyperhomocystinemia are the key risk factors of intracranial stenosis and damages in AIS patients [9, 10]. High-density lipoprotein cholesterol is one of the protective factors of atherosclerosis in ischemic stroke patients [11]. In addition, aspirin is the most commonly used antiplatelet drug and may provide greater protection against the ischemic stroke. We focused our attention on the effects of aspirin and removed the interference of aspirin therapy between the two groups. All the vascular risk factors were excluded. We 
found that stroke was still more serious in the PTA group, despite elevated levels of high-density lipoprotein cholesterol, which further suggested and illustrated the possible specific effects of autoimmunity related to elevated TPO$\mathrm{Ab}$ or $\mathrm{Tg}-\mathrm{Ab}$ on stroke severity in euthyroid state. Although the precise mechanism related to the poor functional outcome is not discussed in this study, the most likely and reasonable speculation is that the vascular damage associated with inappropriate autoimmune response may aggravate stroke severity by contributing to endothelial dysfunction and atherosclerosis [12]. The previous research reported the impairment of endothelial-dependent arterial dilatation in autoimmune thyroiditis patients with euthyroidism, indicating that thyroid autoimmunity may cause endothelial dysfunction [13]. In addition, the endothelial dysfunction was a marker of atherosclerosis risk [14]. Furthermore, brain perfusion in patients with autoimmune thyroiditis was decreased, which suggested the possible relationship between elevated thyroid autoantibodies and unfavorable brain perfusion in AIS [15]. Now that the endothelial dysfunction is recognized as an important early event in atherogenesis, further studies may be needed to clarify the relationship between the thyroid autoantibodies and endothelial dysfunction in AIS patients with normal thyroid function states.

Our study revealed more female than male patients in the group with positive thyroid autoantibodies in China, which was consistent with a previous study that indicated a clear female preponderance with a high prevalence of thyroid autoantibodies in the general population [16-19]. The ratio of female and male ranged from $1.8: 1$ to $4.4: 1$ in the above studies. Our study showed the ratio of female and male was 1:1 in the PTA group; however, the ratio of female and male was $1: 2.3$ in the NTA group. This significant difference between the two groups highlighted an obvious female preponderance. These monoclonal antibodies specific to estrogen receptors and progesterone receptors were present in the thyroid gland tissues [20]. Hence, there could be an association between thyroid autoimmunity reactions and sex hormone receptors. A study showed an independent association of elevated TPO-Ab with intracranial large artery damages in younger stroke patients with normal thyroid function. However, in that case, the independent relationship of elevated $\mathrm{Tg}-\mathrm{Ab}$ on intracranial large artery stenosis could not be found [21]. The levels of Tg-Ab and TPO-Ab increase independently in response to thyroglobulin and thyroid peroxidase, respectively. The prevalence of positive $\mathrm{TPO}-\mathrm{Ab}$ ranges from $3.4 \%$ to $13.4 \%$ in the general population. In our study, the prevalence of elevated TPO-Ab and elevated $\mathrm{Tg}$ - $\mathrm{Ab}$ was $8.8 \%$ and $8.1 \%$, respectively. To investigate thyroid autoimmunity, we included both thyroid autoantibodies to elucidate their effects on functional outcome in patients with AIS.

Our data suggested that lower FT3 values within the normal reference range on admission in the elevated thyroid autoantibodies group may predict a poor functional outcome in AIS patients. Low T3 syndrome is known as euthyroid sick syndrome, which shows a reduction of serum T3 without the elevation of serum TSH [22]. In our study, the mean values of TSH, TT3, TT4, FT3, and FT4 were all within the euthyroid reference range. However, the lower FT3 has been described as being consistent with worse AIS severity. FT3 is generally more sensitive than TT3. The change trend of FT3 can still predict the stroke severity in AIS, even though FT3 is in the normal range.

The limitations of our study include the retrospective nature of our study and the limitation of a single academic institution. We need to further carry out large-scale prospective and multicenter studies to validate our findings.

\section{Conclusions}

Our results indicate that the elevated thyroid autoantibodies aggravate stroke severity in euthyroidism with AIS in Chinese patients. Our findings remind us that we need to further evaluate the role of the autoimmune factors in the pathogenesis of AIS, independent of thyroid function.

\section{Data Availability}

The data used to support the findings of this study are included within the article.

\section{Conflicts of Interest}

The authors declare that there are no conflicts of interest regarding the publication of this paper.

\section{References}

[1] Y. Yoshida, T. Hiwasa, and T. Machida, "Elevation of autoantibody in patients with ischemic stroke," Neurologia MedicoChirurgica (Tokyo), vol. 58, no. 7, pp. 303-310, 2018.

[2] B. Gini, L. Lovato, R. Cianti et al., "Novel autoantigens recognized by CSF IgG from Hashimoto's encephalitis revealed by a proteomic approach," Journal of Neuroimmunology, vol. 196, no. 1-2, pp. 153-158, 2008.

[3] K. Moodley, J. Botha, D. M. Raidoo, and S. Naidoo, "Immunolocalisation of anti-thyroid antibodies in adult human cerebral cortex," Journal of the Neurological Sciences, vol. 302, no. 1-2, pp. 114-117, 2011.

[4] X. Zhang, Z. Chen, Z. Shi, and M. Lou, "Correlation between thyroid autoantibodies and intracranial arterial stenosis in stroke patients with hyperthyroidism," Journal of the Neurological Sciences, vol. 318, no. 1-2, pp. 82-84, 2012.

[5] H. Li, Z. S. Zhang, Z. N. Dong et al., "Increased thyroid function and elevated thyroid autoantibodies in pediatric patients with moyamoya disease: a case-control study," Stroke, vol. 42, no. 4, pp. 1138-1139, 2011.

[6] H. J. Cho, S. S. Kim, S. M. Sung, and D. S. Jung, "Impact of thyroid autoantibodies on functional outcome in patients with acute ischemic stroke," Journal of Stroke and Cerebrovascular Diseases, vol. 23, no. 7, pp. 1915-1920, 2014.

[7] F. A. Wollenweber, V. Zietemann, A. Gschwendtner, C. Opherk, and M. Dichgans, "Subclinical hyperthyroidism is a risk factor for poor functional outcome after ischemic stroke," Stroke, vol. 44, no. 5, pp. 1446-1448, 2013.

[8] U. Utku, T. Asil, Y. Celik, and D. Tucer, "Reversible MR angiographic findings in a patient with autoimmune Graves disease," AJNR. American Journal of Neuroradiology, vol. 25, no. 9, pp. 1541-1543, 2004. 
[9] F. Z. Caprio and F. A. Sorond, "Cerebrovascular disease: primary and secondary stroke prevention," The Medical Clinics of North America, vol. 103, no. 2, pp. 295-308, 2019.

[10] S. G. Chrysant and G. S. Chrysant, "The current status of homocysteine as a risk factor for cardiovascular disease: a mini review," Expert Review of Cardiovascular Therapy, vol. 16, no. 8, pp. 559-565, 2018.

[11] X. Zheng, N. Zeng, A. Wang et al., "Elevated C-reactive protein and depressed high-density lipoprotein cholesterol are associated with poor function outcome after ischemic stroke," Current Neurovascular Research, vol. 15, no. 3, pp. 226-233, 2018.

[12] A. Katsargyris, S. Tsiodras, S. Theocharis et al., "Toll-like receptor 4 immunohistochemical expression is enhanced in macrophages of symptomatic carotid atherosclerotic plaques," Cerebrovascular Diseases, vol. 31, no. 1, pp. 29-36, 2011.

[13] G. Xiang, Y. S. He, L. S. Zhao, J. Hou, L. Yue, and H. J. Xiang, "Impairment of endothelium-dependent arterial dilation in Hashimoto's thyroiditis patients with euthyroidism," Clinical Endocrinology, vol. 64, no. 6, pp. 698-702, 2006.

[14] S. Sitia, L. Tomasoni, F. Atzeni et al., "From endothelial dysfunction to atherosclerosis," Autoimmunity Reviews, vol. 9, no. 12, pp. 830-834, 2010.

[15] M. Piga, A. Serra, L. Deiana et al., "Brain perfusion abnormalities in patients with euthyroid autoimmune thyroiditis," European Journal of Nuclear Medicine and Molecular Imaging, vol. 31, no. 12, pp. 1639-1644, 2004.

[16] M. Kabelitz, K. P. Liesenkötter, B. Stach et al., “The prevalence of anti-thyroid peroxidase antibodies and autoimmune thyroiditis in children and adolescents in an iodine replete area," European Journal of Endocrinology, vol. 148, no. 3, pp. 301-307, 2003.

[17] J. G. Hollowell, N. W. Staehling, W. D. Flanders et al., "Serum TSH, T (4), and thyroid antibodies in the United States population (1988 to 1994): National Health and Nutrition Examination Survey (NHANES III)," The Journal of Clinical Endocrinology, vol. 87, no. 2, pp. 489-499, 2002.

[18] K. Kasagi, N. Takahashi, G. Inoue, T. Honda, Y. Kawachi, and Y. Izumi, "Thyroid function in Japanese adults as assessed by a general health checkup system in relation with thyroid-related antibodies and other clinical parameters," Thyroid, vol. 19, no. 9, pp. 937-944, 2009.

[19] Y. Li, D. Teng, Z. Shan et al., "Antithyroperoxidase and antithyroglobulin antibodies in a five-year follow-up survey of populations with different iodine intakes," The Journal of Clinical Endocrinology, vol. 93, no. 5, pp. 1751-1757, 2008.

[20] S. R. Money, W. Muss, W. L. Thelmo et al., "Immunocytochemical localization of estrogen and progesterone receptors in human thyroid," Surgery, vol. 106, no. 6, pp. 975-8; discussion 979, 1989.

[21] Z. Shi, X. Zhang, Z. Chen, D. S. Liebeskind, and M. Lou, "Elevated thyroid autoantibodies and intracranial stenosis in stroke at an early age," International Journal of Stroke, vol. 9, no. 6, pp. 735-740, 2014.

[22] L. J. De Groot, "Dangerous dogmas in medicine: the nonthyroidal illness syndrome," The Journal of Clinical Endocrinology, vol. 84, no. 1, pp. 151-164, 1999. 